\title{
Quality of life correlation with socioeconomic status in Korean hepatitis-B patients: a cross sectional study
}

\author{
Seung Ju Kim ${ }^{1,2}$, Kyu-Tae Han ${ }^{1,2}$, Seo Yoon Lee ${ }^{2,3}$ and Eun-Cheol Park ${ }^{2,4^{*}}$
}

\begin{abstract}
Background: In Korea, more than two-thirds of hepatocellular carcinoma patients are hepatitis B virus (HBV) surface antigen-seropositive. The effects of HBV infection on health-related quality of life (HRQoL) are important aspects in the overall management of HBV infection. However, other effects of other parameters on HBV patient HRQoL remain unknown and require clarification. Our study evaluated HRQoL in hepatitis-B patients, according to socioeconomic status.

Methods: We used community health survey data to analyze the relationship between HRQoL of $\mathrm{HBV}^{+}$patients according to socioeconomic status. We used propensity score matching (Ratio $=1: 5$ ) to balancing the patients and general population. Final analytic sample consisted of 7,098 hepatitis B patients and compared group (35,490 general populations). We examined the HRQoL in $\mathrm{HBV}^{+}$patients $(n=7,098)$, stratified by socioeconomic status, compared with general populations, using the EuroQOL visual analog scale (EQ-VAS) and EQ-5D questionnaires. We used the Chi-square test and ANOVA to compare demographic variables. Multiple linear regression analysis identified associations between demographic variables and HRQoL.
\end{abstract}

Results: Participants with hepatitis B numbered 7,098 (16.7 \%) of the study population. HRQoL was lower in hepatitis-B patients compared to the general population (EQ-VAS: $-0.985, p=0.0004$; EQ-5D: $-0.673, p=0.0003$ ). According to occupation type, clerks (EQ-VAS: $-2.628, p=0.0030$; EQ-5D: $-0.802, p=0.0099$ ) and managers and professionals (EQ-VAS: $-1.518, p=0.0356$ ) had the lowest HRQoLs. Higher family income and education level groups had lower HRQoLs compared to the general population.

Conclusions: Patients from higher socioeconomic status groups had HRQoLs that were more affected by hepatitis B. Thus, we require more accurate information about the disease to develop appropriate patient management guidelines. This will facilitate formulating policies and management strategies that alleviate HRQoL declines in $\mathrm{HBV}^{+}$patients.

\section{Background}

The global burden of chronic diseases contributes to a major public health challenge that undermines social and economic development worldwide. An estimated 39 million deaths occurred globally in 2008 that were due to noncommunicable diseases such as cardiovascular disease $(48 \%)$, cancer (21\%), chronic respiratory disease $(12 \%)$, and diabetes (3.5\%) [1]. Globally, of these diseases, cancer is increasingly responsible for a large proportion of

\footnotetext{
* Correspondence: ecpark@yuhs.ac

${ }^{2}$ Institute of Health Services Research, Yonsei University, Seoul, Republic of Korea

${ }^{4}$ Department of Preventive Medicine, Yonsei University College of Medicine, 50 Yonsei-ro, Seodaemun-gu, Seoul 120-752, Republic of Korea

Full list of author information is available at the end of the article
}

deaths, and two of the most common sites for mortality are the liver and stomach [2].

In Korea, cancer is the leading cause of mortality, and the liver is second most common primary tumor site that causes death. Liver cancer prevalence in Korea also increased during a recent 10-year span, from $29.0 \%$ in 2001 to 32.9 \% in 2011 [3]. Hepatitis B virus (HBV) infection is a leading risk factor for developing hepatocellular carcinoma globally, and in Korea more than two-thirds $(74.2 \%)$ of liver cancer cases tested positive for circulating HBV surface antigen [4]. The most important approach to preventing HBV infection is childhood vaccination, and the World Health Organization recommended that all countries introduce a policy of universal HBV vaccination in 1991; as of 2011, this policy had been introduced 
nationwide in 180 countries, with coverage approaching $80 \%$ [5]. Regardless of the mode of infection, HBV infection remains highly endemic in the Western Pacific region $[6,7]$. In the Korean general population, the HBV surface antigen-positive prevalence rate has decreased from $10 \%$ in the 1980 s to $3.8 \%$ in 2007 , following the introduction of HBV vaccination [8]. However, a previous study suggested that this decreased HBV prevalence is limited to the young population and that HBV infection persists in the middle-aged and older populations [9]. And HBV seems to have more important role than hepatitis $C$ in Korea, with respect to promoting the pathogenesis of specific hematologic malignancies, and is associated with increased risk of lymphomas and acute myeloid leukemia [10]. Thus, preventing and managing HBV infection are important considerations in Korean public health.

Measuring health-related quality of life (HRQoL) is commonly used to gauge outcomes in many other diseases. HRQoL is often linked to health, and components of happiness and satisfaction with life are emphasized. The impact of liver disease on HRQoL is multifaceted. In general, the presence of liver disease seems to negatively affect HRQoL. Previous studies identified a close association between hepatitis $\mathrm{C}$, depression, and HRQoL [11-16]. Also, the association between HBV infection and quality of life was documented [17-21]. Despite increasing the interest in assessing HRQoL in different types of liver disease, research into other factors that can affect HRQoL in $\mathrm{HBV}^{+}$patients is lacking. Not only is there a need for studies on general aspects of HBV and HRQoL, but there is also need for assessing the effects of socioeconomic status on patient HRQoL. Especially, Korea has traditional values and unique working environments that may affect patient HRQoL [22]. Our study evaluated the HRQoL associated with hepatitis B patients using a visual analogue scale and the EQ-5D survey. We further explored HRQoL differences in HBV patients according to socioeconomic status.

\section{Methods}

\section{Study population}

The data used in this study were obtained from the 2011-2012 Community Health Survey administered by the Korea Centers for Disease Control and Prevention, which was designed to facilitate interprovincial comparisons. The Community Health Survey was administered by investigators who conducted one-on-one visits and interviews targeting adults $\geq 19$-years-old in 253 health centers nationwide, starting in 2008. We excluded hepatitis $C$ patients and participants with incomplete HRQoL information; $\mathrm{HBV}+$ patients were defined as people who were ever diagnosed with hepatitis $B$, and those histories of $\mathrm{HBV}+$ in people were investigated by interview.
In the data used in our study, only $2.5 \%$ of participants had hepatitis $B$, in order to achieve fair comparison, it was necessary to balancing the patients and general population. Thus, we refer to the two groups as the hepatitis B patients and comparison groups and assume that a patient is matched to one or more comparison general population. Propensity score matching ( Ratio $=1: 5)$ was used in our paper by matching participants on a number of covariates that may affect in each participant group. This approach is that participants have an underlying propensity to be in one group or another. By matching based on preexisting characteristics, provides a way of statistically controlling the variation in these characteristics to minimize selection effects. A total analytic sample consisted of 7,098 hepatitis B patients and compared group (35,490 general populations) came from similar characteristics after matching the propensity score.

\section{Variables}

The outcome variables were evaluated using the EuroQOL visual analog scale (EQ-VAS) and EQ-5D. The EQ-VAS is a self-rated health questionnaire presented as a vertical visual analog scale, where the endpoints are labeled 'best imaginable health state' and 'worst imaginable health state'. Participants completed the questionnaires on the study day. Scores ranged from 0 to 100 and the responses were used as a quantitative measure of participant selfrated health. The EQ-5D is a questionnaire that characterized health in five domains, i.e., mobility, self-care, usual activities, pain/discomfort, and anxiety/depression on three levels to define HRQoL values.

Other independent variables considered in the analysis were occupation type, marital status, sex, smoking status, alcohol consumption, stress, number of chronic disease, education, income, age, and year. Occupations were divided into managers and professionals, clerks, service and sales workers, skilled agricultural, forestry and fishery workers, trade workers and elementary occupation, and not working. Marital status was classified as "Single", "Separated/Divorced/Bereavement" or "Married", and smoking status as "Smoker", "Ex-smoker", or "Nonsmoker". Alcohol consumption was defined as "Yes" or "No" following the question: "Have you ever drank alcohol in the past one year?" Stress was classified as those who reported feeling stress or those who did not. Chronic disease classified to three groups according to a number of disease which was included asthma, diabetes, hypertension, dyslipidemia and thyroid lesion. People who do not have these chronic diseases were classified as ' 0 '. All disease was defined as people who ever had been diagnosed each disease. Education level was classified as elementary school, middle school, high school, or college graduate. Family income was classified into four groups based on quartiles (Q1: lowest 
income/Q4: highest income), and age was classified into 19-29 years, 30-39 years, 40-49 years, 50-59 years, and $\geq 60$-years-old.

\section{Statistical analyses}

All analyses were conducted using SAS version 9.3 (SAS Institute Inc., Cary, NC). We assigned weight to sampling results to convey an accurate representation of the whole nation. Baseline demographic and clinical characteristics were compared using the Chi-squared test. We next compared the average EQ-VAS and EQ-5D scores according to the independent variables, using ANOVA. In the fully adjusted model, all variables were entered simultaneously. Multiple linear regression analysis was performed to identify associations of these variables with HRQoL (normal population vs hepatitis B), while controlling for potential confounding variables. Additionally, we performed subgroup analyses based on occupation, education, and family income. P-values $<0.05$ were considered indicative of statistically significant differences.

\section{Results}

In our study, a total of 7098 (16.7 \%) of participants were $\mathrm{HBV}$-positive. In occupation type, not working $(33.4 \%)$ and trade workers and elementary occupations (24.6\%) comprised the highest $\mathrm{HBV}^{+}$frequencies. A total of $830(11.7 \%) \mathrm{HBV}^{+}$participants were single, 982 $(13.8 \%)$ participants were separated, divorced or bereaved, and 5,286 (74.4 \%) were married. And male participants that were $\mathrm{HBV}^{+}(\mathrm{n}=3,947 ; 55.6 \%)$ were frequent than females $(n=3,151 ; 44.4 \%)$. Regarding smoking status, almost half of $\mathrm{HBV}^{+}$participants were non-smokers 3,771 (53.1 \%), smokers numbered 1,798 (25.3\%), and exsmokers were 1,529 (21.5 \%). More participants with HBV were alcohol consumers $(\mathrm{n}=4,844 ; 68.2 \%)$ than nonconsumers $(\mathrm{n}=2,254 ; 31.8 \%)$. Of $\mathrm{HBV}^{+}$participants, 2,216 (31.2 \%) reported feeling stressed. Almost twothirds of participants $(\mathrm{n}=4,357 ; 61.4 \%)$ didn't report concurrent chronic diseases (Table 1).

The average EQ-VAS and EQ-5D scores were higher in general population than in the $\mathrm{HBV}^{+}$group $\left(\mathrm{HBV}^{+}\right.$: EQ-VAS: 70.2; EQ-5D: 93.1/general population: EQVAS: 71.2; EQ-5D: 93.8). Regarding occupation type, the managers and professionals category scored highest on the EQ-VAS and EQ-5D, in both the $\mathrm{HBV}^{+}$group and in the general population. Regarding marital status, 'single' scored highest in both the $\mathrm{HBV}^{+}$group and in the general population. As education increased, EQ-VAS and EQ-5D scores gradually increased. A similar trend was found in the family income analyses, in which high income family had higher HRQoL scores in both the $\mathrm{HBV}^{+}$group and in the general population, but scores were higher in the general population (Table 2).
In regression model analysis, $\mathrm{HBV}^{+}$patients had lower EQ-VAS $(-0.985, \mathrm{p}=0.0004)$ and EQ-5D $(-0.673, \mathrm{p}<$ 0.0001 ) scores than the general population. Quality of life scores were lower in low academic status (EQVAS: -4.869, p < 0.0001; EQ-5D: -4.928, p<0.0001). A similar trend was found in family income, wherein lower family income has the lowest HRQoL scores (EQ-VAS: -5.889, $\mathrm{p}<0.0001$; EQ-5D: $-2.355, \mathrm{p}<0.0001$ ). According to age, younger people scored highest on the EQ-5D (4.004, $\mathrm{p}<0.0001)$ referring to people aged $\geq 60$ years-old. According to occupation, managers and professionals scored highest on quality of life compared to not-working participants (EQ-VAS: 3.418, $\mathrm{p}<0.0001$; EQ-5D: 3.518, $\mathrm{p}<0.0001)$. Regarding marital status, separated, divorced, and bereaved participants had lower scores on the EQ-VAS $(-2.395, \mathrm{p}<0.0001)$ and EQ-5D $(-2.018, \mathrm{p}<0.0001)$ compared to married participants (Table 3).

Also, to compare $\mathrm{HBV}^{+}$participants with general population in each variable group, we performed subgroup analyses by considering each variable such as occupation type, education, and family income. In the results of sub-group analysis by occupation types, clerks scored the lowest on the EQ-VAS $(-2.628, \mathrm{p}=0.0030)$, followed by managers and professionals $(-1.518, \mathrm{p}=0.0356)$. Similar results were observed with the EQ-5D, on which trade workers and elementary occupations $(-0.828, \mathrm{p}=0.0007)$, following by clerks $(-0.802, \mathrm{p}=0.0099)$ and not-working $(-0.743, \mathrm{p}=0.0251)$. Regarding educational level, $\mathrm{HBV}^{+}$ patients that were college graduates had the lowest score on the EQ-VAS $(-1.606, \mathrm{p}=0.0003)$ high school graduates scored lowest on the EQ-5D $(-0.968, \mathrm{p}<0.0001)$. There was a trend of decreased EQ-VAS scores in participants with higher academic status. According to family income, quartile-1 (Q1) $\mathrm{HBV}^{+}$patients had lowest EQ-VAS scores $(-1.302, \mathrm{p}=0.0683)$ but, it is not statistically significant. Q2 and Q4 patients scored the lowest on the EQ-5D (Q2: -0.681, $\mathrm{p}=0.0155 ; \mathrm{Q} 4:-0.872, \mathrm{p}=0.0003)$ (Table 4).

\section{Discussion}

Because an estimated 240 million people worldwide are chronically infected with HBV, it is important to understand the effects of this disease on patient health and HRQoL [5]. With growing awareness of the implications of chronic liver disease on HRQoL, research has been conducted by several groups into the effects of HBV infection on patient HRQoL. Assessing the health-utility score reported by these patients would allow us to better understand the effects of $\mathrm{HBV}^{+}$status on these patients QoL and would help to improve their care management.

Our study assessed differences of HRQoL in $\mathrm{HBV}^{+}$patients compared to the general population. The proportion of $\mathrm{HBV}^{+}$patients in our study was $16.7 \%$ of total participants, and $33.4 \%$ of the infected patients were 
Table 1 General characteristics of participants (N, \%)

\begin{tabular}{|c|c|c|c|c|c|c|}
\hline & \multicolumn{2}{|c|}{ Hepatitis B patients } & \multicolumn{2}{|c|}{ General population } & Total & p-value \\
\hline \multicolumn{7}{|l|}{ Occupation } \\
\hline Managers and professionals & 781 & $(11.0)$ & 3901 & $(11.0)$ & 4,682 & \multirow[t]{6}{*}{0.6203} \\
\hline Clerks & 590 & $(8.3)$ & 2,955 & $(8.3)$ & 3,545 & \\
\hline Service and sales workers & 1,024 & $(14.4)$ & 5,254 & $(14.8)$ & 6,278 & \\
\hline Skilled agricultural, forestry and fishery workers & 591 & $(8.3)$ & 3,060 & $(8.6)$ & 3,651 & \\
\hline Trade workers and elementary occupations & 1,744 & (24.6) & 8,385 & (23.6) & 10,129 & \\
\hline Not working & 2,368 & $(33.4)$ & 11,935 & (33.6) & 14,303 & \\
\hline \multicolumn{7}{|l|}{ Marital status } \\
\hline Single & 830 & $(11.7)$ & 3,956 & $(11.1)$ & 4,786 & \multirow[t]{3}{*}{0.3920} \\
\hline Seperated/Divorced/Bereavement & 982 & $(13.8)$ & 4,890 & (13.8) & 5,872 & \\
\hline Married & 5,286 & $(74.5)$ & 26,644 & $(75.1)$ & 31,930 & \\
\hline \multicolumn{7}{|l|}{ Sex } \\
\hline Male & 3,947 & $(55.6)$ & 19,778 & $(55.7)$ & 23,725 & \multirow[t]{2}{*}{0.8512} \\
\hline Female & 3,151 & $(44.4)$ & 15,712 & $(44.3)$ & 18,863 & \\
\hline \multicolumn{7}{|l|}{ Smoking status } \\
\hline Smoker & 1,798 & $(25.3)$ & 8,846 & $(24.9)$ & 10,644 & \multirow[t]{3}{*}{0.7434} \\
\hline Ex-smoker & 1,529 & $(21.5)$ & 7,736 & (21.8) & 9,265 & \\
\hline Non-smoker & 3,771 & $(53.1)$ & 18,908 & $(53.3)$ & 22,679 & \\
\hline \multicolumn{7}{|l|}{ Alcohol consumption } \\
\hline Yes & 4,844 & $(68.2)$ & 24,407 & (68.8) & 29,251 & \multirow[t]{2}{*}{0.3822} \\
\hline No & 2,254 & $(31.8)$ & 11,083 & $(31.2)$ & 13,337 & \\
\hline \multicolumn{7}{|l|}{ Stress } \\
\hline Yes & 2,216 & $(31.2)$ & 11,075 & $(31.2)$ & 13,291 & \multirow[t]{2}{*}{0.9813} \\
\hline No & 4,882 & $(68.8)$ & 24,415 & $(68.8)$ & 29,297 & \\
\hline \multicolumn{7}{|l|}{ Chronic disease } \\
\hline 0 & 4,357 & (61.4) & 22,590 & $(63.7)$ & 26,947 & \\
\hline 1 & 1,791 & $(25.2)$ & 8,590 & $(24.2)$ & 10,381 & \\
\hline$\geq 2$ & 950 & $(13.4)$ & 4,310 & $(12.1)$ & 5,260 & \\
\hline \multicolumn{7}{|l|}{ Education } \\
\hline Elementary school & 1,425 & $(20.1)$ & 7,043 & (19.8) & 8,468 & \multirow[t]{4}{*}{0.6587} \\
\hline Middle school & 1,049 & $(14.8)$ & 5,411 & $(15.2)$ & 6,460 & \\
\hline High school graduate & 2,666 & $(37.6)$ & 13,416 & (37.8) & 16,082 & \\
\hline College graduate & 1,958 & $(27.6)$ & 9,620 & (27.1) & 11,578 & \\
\hline
\end{tabular}


Table 1 General characteristics of participants (N, \%) (Continued)

\begin{tabular}{|c|c|c|c|c|c|c|}
\hline \multicolumn{7}{|c|}{ Family income } \\
\hline Q1 & 1,697 & (23.9) & 8,418 & (23.7) & 10,115 & \multirow[t]{4}{*}{0.9870} \\
\hline Q2 & 2,152 & (30.3) & 10,813 & (30.5) & 12,965 & \\
\hline Q3 & 1,953 & $(27.5)$ & 9,765 & (27.5) & 11,718 & \\
\hline Q4 & 1,296 & (18.3) & 6,494 & (18.3) & 7,790 & \\
\hline \multicolumn{7}{|l|}{ Age } \\
\hline $20-29$ & 542 & (7.6) & 2,645 & (7.5) & 3,187 & \multirow[t]{5}{*}{0.9257} \\
\hline $30-39$ & 1,127 & $(15.9)$ & 5,575 & $(15.7)$ & 6,702 & \\
\hline $40-49$ & 1,647 & $(23.2)$ & 8,177 & (23.0) & 9,824 & \\
\hline $50-59$ & 1,834 & (25.8) & 9,191 & (25.9) & 11,025 & \\
\hline $60 \leq$ & 1,948 & (27.4) & 9,902 & (27.9) & 11,850 & \\
\hline \multicolumn{7}{|l|}{ Year } \\
\hline 2011 & 3,578 & (50.4) & 17,917 & (50.5) & 21,495 & \multirow[t]{2}{*}{0.9068} \\
\hline 2012 & 3,520 & $(49.6)$ & 17,573 & $(49.5)$ & 21,093 & \\
\hline Total & 7,098 & $(16.7)$ & 35,490 & (83.3) & 42,588 & $<.0001$ \\
\hline
\end{tabular}


Table 2 Relationships of quality of life with demongraphic characteristics

\begin{tabular}{|c|c|c|c|c|c|c|c|c|c|c|c|c|}
\hline & \multicolumn{6}{|c|}{ Hepatitis B patients } & \multicolumn{6}{|c|}{ General population } \\
\hline & \multicolumn{3}{|c|}{ EQ-VAS } & \multicolumn{3}{|l|}{$E Q-5 D$} & \multicolumn{3}{|l|}{ EQ-VAS } & \multicolumn{3}{|l|}{ EQ-5D } \\
\hline & Means & SD & P-Value & Means & SD & P-Value & Means & SD & P-Value & Means & SD & P-Value \\
\hline \multicolumn{13}{|l|}{ Occupation } \\
\hline Managers and professionals & 74.9 & 15.3 & $<.0001$ & 97.2 & 6.1 & $<.0001$ & 76.2 & 14.8 & $<.0001$ & 97.9 & 5.4 & $<.0001$ \\
\hline Clerks & 73.1 & 17.3 & & 97.1 & 6.6 & & 75.3 & 14.8 & & 98.2 & 5.1 & \\
\hline Service and sales workers & 72.5 & 16.5 & & 95.7 & 7.3 & & 73.2 & 16.6 & & 96.3 & 7.6 & \\
\hline Skilled agricultural, forestry and fishery workers & 67.7 & 18.0 & & 91.1 & 12.8 & & 68.8 & 18.3 & & 91.9 & 11.2 & \\
\hline Trade workers and elementary occupations & 71.6 & 17.3 & & 95.3 & 8.6 & & 73.0 & 16.8 & & 96.3 & 7.8 & \\
\hline Not working & 66.6 & 20.9 & & 88.5 & 14.9 & & 67.1 & 20.1 & & 88.9 & 15.4 & \\
\hline \multicolumn{13}{|l|}{ Marital status } \\
\hline Single & 71.3 & 17.8 & 0.0107 & 95.9 & 9.0 & $<.0001$ & 73.7 & 16.9 & $<.0001$ & 96.8 & 8.7 & $<.0001$ \\
\hline Seperated/Divorced/Bereavement & 64.5 & 20.5 & & 87.3 & 15.0 & & 64.8 & 20.0 & & 87.9 & 15.1 & \\
\hline Married & 71.1 & 18.1 & & 93.7 & 10.9 & & 72.1 & 17.5 & & 94.4 & 10.8 & \\
\hline \multicolumn{13}{|l|}{ Sex } \\
\hline Male & 71.6 & 18.0 & 0.0107 & 94.6 & 10.7 & 0.0002 & 72.7 & 17.4 & $<.0001$ & 95.0 & 10.9 & $<.0001$ \\
\hline Female & 68.5 & 19.1 & & 91.2 & 12.3 & & 69.4 & 18.6 & & 92.2 & 12.2 & \\
\hline \multicolumn{13}{|l|}{ Smoking status } \\
\hline Smoker & 70.0 & 18.4 & 0.0054 & 94.3 & 11.0 & 0.1629 & 70.9 & 17.8 & $<.0001$ & 95.2 & 10.2 & 0.0005 \\
\hline Ex-smoker & 71.5 & 18.5 & & 93.5 & 11.3 & & 72.4 & 18.0 & & 93.5 & 12.6 & \\
\hline Non-smoker & 69.9 & 18.7 & & 92.3 & 11.9 & & 70.9 & 18.1 & & 93.2 & 11.6 & \\
\hline \multicolumn{13}{|l|}{ Alcohol consumption } \\
\hline Yes & 71.5 & 17.5 & 0.0079 & 94.5 & 10.0 & 0.0027 & 72.7 & 17.0 & $<.0001$ & 95.5 & 9.3 & $<.0001$ \\
\hline No & 67.5 & 20.4 & & 90.1 & 14.0 & & 68.1 & 19.7 & & 90.0 & 14.8 & \\
\hline \multicolumn{13}{|l|}{ Stress } \\
\hline Yes & 63.8 & 20.1 & $<.0001$ & 89.8 & 14.2 & $<.0001$ & 65.3 & 19.5 & $<.0001$ & 90.8 & 14.1 & $<.0001$ \\
\hline No & 73.2 & 17.0 & & 94.6 & 9.8 & & 73.9 & 16.6 & & 95.1 & 9.9 & \\
\hline \multicolumn{13}{|l|}{ Chronic disease } \\
\hline 0 & 72.0 & 17.5 & $<.0001$ & 95.0 & 9.6 & $<.0001$ & 73.2 & 17.1 & $<.0001$ & 95.6 & 9.4 & $<.0001$ \\
\hline 1 & 68.9 & 19.5 & & 91.3 & 12.7 & & 68.9 & 18.8 & & 91.5 & 13.4 & \\
\hline$\geq 2$ & 64.6 & 20.2 & & 87.6 & 15.0 & & 65.7 & 19.5 & & 88.5 & 14.8 & \\
\hline
\end{tabular}


Table 2 Relationships of quality of life with demongraphic characteristics (Continued)

\begin{tabular}{|c|c|c|c|c|c|c|c|c|c|c|c|c|}
\hline \multicolumn{13}{|l|}{ Education } \\
\hline Elementary school & 63.3 & 20.0 & $<.0001$ & 85.8 & 15.6 & $<.0001$ & 64.0 & 20.2 & $<.0001$ & 86.2 & 15.5 & $<.0001$ \\
\hline Middle school & 68.4 & 19.5 & & 91.5 & 12.4 & & 69.4 & 18.7 & & 92.0 & 12.7 & \\
\hline High school graduate & 72.1 & 17.8 & & 94.9 & 9.4 & & 72.9 & 17.1 & & 95.8 & 9.3 & \\
\hline College graduate & 73.9 & 16.3 & & 96.7 & 7.2 & & 75.4 & 15.3 & & 97.5 & 6.4 & \\
\hline \multicolumn{13}{|l|}{ Family income } \\
\hline Q1 & 63.2 & 20.5 & $<.0001$ & 86.4 & 15.7 & $<.0001$ & 64.1 & 20.2 & $<.0001$ & 87.2 & 15.5 & $<.0001$ \\
\hline Q2 & 70.2 & 17.9 & & 93.7 & 10.3 & & 71.5 & 17.6 & & 94.3 & 10.6 & \\
\hline Q3 & 73.3 & 16.8 & & 96.1 & 8.0 & & 74.0 & 16.3 & & 96.5 & 7.9 & \\
\hline Q4 & 74.9 & 16.6 & & 96.3 & 7.6 & & 76.0 & 15.3 & & 97.1 & 7.4 & \\
\hline \multicolumn{13}{|l|}{ Age } \\
\hline $20-29$ & 73.3 & 17.7 & 0.0202 & 97.5 & 6.0 & $<.0001$ & 75.3 & 16.4 & $<.0001$ & 98.0 & 5.6 & $<.0001$ \\
\hline $30-39$ & 71.3 & 17.3 & & 96.4 & 7.8 & & 73.5 & 16.0 & & 97.7 & 5.7 & \\
\hline $40-49$ & 72.9 & 17.2 & & 95.6 & 8.7 & & 73.5 & 16.7 & & 96.5 & 8.2 & \\
\hline 50-59 & 70.6 & 18.8 & & 93.3 & 11.2 & & 71.9 & 17.9 & & 94.3 & 10.9 & \\
\hline $60 \leq$ & 66.2 & 19.6 & & 87.6 & 14.7 & & 66.4 & 19.7 & & 87.7 & 15.1 & \\
\hline \multicolumn{13}{|l|}{ Year } \\
\hline 2011 & 69.0 & 18.9 & $<.0001$ & 92.8 & 11.9 & 0.0063 & 70.1 & 18.5 & $<.0001$ & 93.4 & 11.9 & $<.0001$ \\
\hline 2012 & 71.5 & 18.1 & & 93.4 & 11.3 & & 72.4 & 17.4 & & 94.1 & 11.2 & \\
\hline Total & 70.2 & 18.6 & & 93.1 & 11.6 & & 71.2 & 18.0 & & 93.8 & 11.5 & \\
\hline
\end{tabular}


Table 3 Regression model analysis results of EQ-VAS and EQ-5D

\begin{tabular}{|c|c|c|c|c|}
\hline & \multicolumn{2}{|l|}{ EQ-VAS } & \multicolumn{2}{|l|}{ EQ-5D } \\
\hline & Estimate & $\mathrm{p}$-value & Estimate & p-value \\
\hline \multicolumn{5}{|l|}{ Hepatitis B } \\
\hline Yes & -0.985 & 0.0004 & -0.673 & $<.0001$ \\
\hline No & Ref & - & Ref & - \\
\hline \multicolumn{5}{|l|}{ Occupation } \\
\hline Managers and professionals & 3.418 & $<.0001$ & 3.518 & $<.0001$ \\
\hline Clerks & 2.911 & $<.0001$ & 3.421 & $<.0001$ \\
\hline Service and sales workers & 2.809 & $<.0001$ & 3.552 & $<.0001$ \\
\hline Skilled agricultural, forestry and fishery workers & 2.048 & $<.0001$ & 3.793 & $<.0001$ \\
\hline Trade workers and elementary occupations & 2.746 & $<.0001$ & 3.950 & $<.0001$ \\
\hline Not working & Ref & - & Ref & - \\
\hline \multicolumn{5}{|l|}{ Marital status } \\
\hline Single & -0.486 & 0.219 & -0.497 & 0.0073 \\
\hline Seperated/Divorced/Bereavement & -2.395 & $<.0001$ & -2.018 & $<.0001$ \\
\hline Married & Ref & - & Ref & - \\
\hline \multicolumn{5}{|l|}{ Sex } \\
\hline Male & 2.245 & $<.0001$ & 0.690 & $<.0001$ \\
\hline Female & Ref & - & Ref & - \\
\hline \multicolumn{5}{|l|}{ Smoking status } \\
\hline Smoker & -2.870 & $<.0001$ & -0.372 & 0.0179 \\
\hline Ex-smoker & -0.606 & 0.0711 & -0.271 & 0.1316 \\
\hline Non-smoker & Ref & - & Ref & - \\
\hline \multicolumn{5}{|l|}{ Alcohol consumption } \\
\hline Yes & 0.937 & 0.0002 & 1.399 & $<.0001$ \\
\hline No & Ref & - & Ref & - \\
\hline \multicolumn{5}{|l|}{ Stress } \\
\hline Yes & -8.368 & $<.0001$ & -4.105 & $<.0001$ \\
\hline No & Ref & - & Ref & - \\
\hline \multicolumn{5}{|l|}{ Chronic disease } \\
\hline 0 & Ref & - & Ref & - \\
\hline 1 & -2.150 & $<.0001$ & -1.213 & $<.0001$ \\
\hline$\geq 2$ & -4.617 & $<.0001$ & -2.848 & $<.0001$ \\
\hline
\end{tabular}


Table 3 Regression model analysis results of EQ-VAS and EQ-5D (Continued)

\begin{tabular}{|c|c|c|c|c|}
\hline \multicolumn{5}{|l|}{ Education } \\
\hline Elementary school & -4.869 & $<.0001$ & -4.928 & $<.0001$ \\
\hline Middle school & -3.003 & $<.0001$ & -2.500 & $<.0001$ \\
\hline High school graduate & -0.786 & 0.0037 & -0.588 & $<.0001$ \\
\hline College graduate & Ref & - & Ref & - \\
\hline \multicolumn{5}{|l|}{ Family income } \\
\hline Q1 & -5.889 & $<.0001$ & -3.587 & $<.0001$ \\
\hline Q2 & -2.355 & $<.0001$ & -0.825 & $<.0001$ \\
\hline Q3 & -1.278 & $<.0001$ & -0.340 & 0.0077 \\
\hline Q4 & Ref & - & Ref & - \\
\hline \multicolumn{5}{|l|}{ Age } \\
\hline $20-29$ & 0.914 & 0.1106 & 4.004 & $<.0001$ \\
\hline $30-39$ & -1.253 & 0.0032 & 3.044 & $<.0001$ \\
\hline $40-49$ & -0.440 & 0.2496 & 2.269 & $<.0001$ \\
\hline $50-59$ & 0.280 & 0.4208 & 1.983 & $<.0001$ \\
\hline $60 \leq$ & Ref & - & Ref & - \\
\hline \multicolumn{5}{|l|}{ Year } \\
\hline 2011 & -2.056 & $<.0001$ & -0.431 & 0.0001 \\
\hline 2012 & Ref & - & Ref & - \\
\hline
\end{tabular}


Table 4 Sub-group analysis of EQ-VAS and EQ-5DS scale according to occupation, education and family income level in hepatitis B (unit: coefficient, $p=$ value)

\begin{tabular}{|c|c|c|c|c|}
\hline & \multicolumn{2}{|l|}{ EQ-VAS } & \multicolumn{2}{|l|}{ EQ-5D } \\
\hline & Estimate* & $p$-value & Estimate* & $p$-value \\
\hline \multicolumn{5}{|l|}{ Occupation } \\
\hline Managers and professionals & -1.518 & 0.0356 & -0.582 & 0.0209 \\
\hline Clerks & -2.628 & 0.0030 & -0.802 & 0.0099 \\
\hline Service and sales workers & -0.529 & 0.4362 & -0.663 & 0.0217 \\
\hline Skilled agricultural, forestry and fishery workers & 0.162 & 0.8720 & -1.063 & 0.1168 \\
\hline Trade workers and elementary occupations & -0.654 & 0.2373 & -0.828 & 0.0007 \\
\hline Not working & -0.937 & 0.069 & -0.743 & 0.0251 \\
\hline \multicolumn{5}{|l|}{ Education } \\
\hline Elementary school & -0.127 & 0.8652 & -0.747 & 0.1832 \\
\hline Middle school & -0.471 & 0.5298 & -0.333 & 0.482 \\
\hline High school graduate & -0.952 & 0.0407 & -0.968 & $<.0001$ \\
\hline College graduate & -1.606 & 0.0003 & -0.532 & 0.0047 \\
\hline \multicolumn{5}{|l|}{ Family income } \\
\hline Q1 & -1.302 & 0.0683 & -0.910 & 0.0587 \\
\hline Q2 & -1.106 & 0.0368 & -0.681 & 0.0155 \\
\hline Q3 & -0.732 & 0.1351 & -0.371 & 0.0934 \\
\hline Q4 & -0.884 & 0.1077 & -0.872 & 0.0003 \\
\hline
\end{tabular}

*All coefficients are adjuted for marital status, sex, smoking status, alcohol consumption, stress, number of chronic diseases, age and year *All coefficients are the results of hepatitis B patients compared to general population

not working. We also found that $68.2 \%$ of $\mathrm{HBV}^{+}$patients consumed alcohol, which was similar to the $68.8 \%$ drinking rate in the general population. These results might be considered with respect to patient employment status. In Korea, many employees informally gather to drink alcohol in coworker networks to promote working relationships [23]. This cultural trait might be affected alcohol consumption in both of general population and HBV patients.

In our study, $\mathrm{HBV}^{+}$patients scored lower in HRQoL parameters than did the general population. This observation is likely related to the disease status, wherein HBV patients feel discomfort and fatigue during everyday life activities, which might negatively affect their mental health. These results were similar to those of previous studies on the association between HBV status and patient HRQoL [18, 24-26]. However, we also investigated several additional factors that could potentially affect HRQoL, such as education, family income, and occupation.

In sub-group analysis, according to occupation type, clerks had the lowest scores in EQ-VAS which were even lower than scores of not-working patients. These results contrast with other findings of lowest HRQoL scores in unemployed $\mathrm{HBV}^{+}$patients [27]. This disparity might be due to specific occupational characteristics [28]. Clerks usually deal directly with customers and may feel more emotional stress than other occupations. Because they need to accommodate customer complaints and satisfy supervisor demands, these occupations may have inherently higher stress levels that negatively affect their HRQoL. Also, because working schedules in these occupations are generally inflexible, these patients might not be able to visit the hospital even if they felt sick. Inability to seek timely medical care might further negatively affect these HBV patients' HRQoL.

Hepatitis-B patients with higher education status had lower HRQoL scores compared to the general population. This might be a result of the social climate in Korea, in which generally it is difficult to people to have sick leave, even though they are cancer [22, 29]. Because, chronic hepatitis B is a disease with no specific visible symptoms, it might be considered a fake illness to other people. If $\mathrm{HBV}^{+}$patients take sick leave, they might encounter social stigmatization because other people may think that these patients were not working faithful and hard. Eventually, this belief might affect $\mathrm{HBV}^{+}$patients' promotion and they could be excluded from important positions. In this way, the social mood in Korea might preferentially and negatively affect $\mathrm{HBV}^{+}$patients with higher academic status.

We found that high-income $\mathrm{HBV}^{+}$patients had lower HRQoL scores on the EQ-5D survey. Usually, higherincome status would be had more opportunity to management of themselves, through visiting hospital or maintaining good nutrition. However, chronic hepatitis 
could be lead to liver cancer in the future, it might be affected negatively to patients due to the un-awareness of their disease progression.

This study had limitations. First, it was a crosssectional design; therefore, causal relationships between $\mathrm{HBV}^{+}$patient HRQoL and socioeconomic status factors could not be definitively established. Second, we did not consider disease progression and severity in HBV+ patients; therefore, the results may not be applicable some patients such as those with resultant cirrhosis or liver cancer. Also, we only considered chronic diseases such as asthma, diabetes, hypertension, dyslipidemia and thyroid lesion because the data used in our study not included other chronic diseases. Finally, we did not assess potential changes in patient occupation type and income after HBV diagnosis, which may have impacted HRQoL. Despite these limitations, our study had several strengths. First, we used data from the Community Health Survey, which ensured use of a reliable nationwide sampling design. Second, we analyzed differences in $\mathrm{HRQoL}$ in $\mathrm{HBV}^{+}$patients versus the general population, not only based on HBV status, but also include according to the type of occupation, education history, and family income. The results of our subgroup analyses might help us to better manage $\mathrm{HBV}^{+}$patient care to improve their HRQoL. Third, we used propensity score matching method, it might be reduce bias in estimating effect of quality of life in the HBV patients compared general population [30].

Our findings indicate that $\mathrm{HBV}^{+}$patients have lower HRQoL scores compared to the general population, especially in patients employed as clerks and managers and professionals, and in those with and higher academic and income statuses. These results highlight the important role of health care providers in managing $\mathrm{HBV}^{+}$patient care and educating the general population with accurate information about the disease [31-33]. Having exact information regarding how HBV status affects patient $\mathrm{HRQOL}$ is critical to understanding disease impact on everyday activities and attitudes. Although HBV does not manifest visible symptoms of illness, patients still must continuously manage themselves to stop the disease from worsening. Education should include not only pathology details of the disease but also the importance of routine disease screening [33]. It is necessary to provide public education and awareness campaigns that play important roles in promoting HBV screening.

Additionally, companies have to increase employee understanding of the disease and provide regular health inspections. This may help to cultivate the knowledge of the disease and reduce prejudices about $\mathrm{HBV}^{+}$patients. And, superiors in the service industries have to consider giving sick leave to $\mathrm{HBV}^{+}$employees so that they can go to the hospital and check their health condition, when necessary.

\section{Conclusions}

In conclusion, we identified significant relationships between $\mathrm{HBV}^{+}$patient $\mathrm{HRQoL}$ and socioeconomic factors such as occupation type, education level, and family income, although further study of these associations is needed. Worldwide efforts to prevent HBV infection have been very successful; now, we have to focus on optimally managing $\mathrm{HBV}^{+}$patient care and improving their HRQoL. To do this, we must acquire accurate information about the disease, including HBV impact on patient lifestyle and sense of well-being. This will facilitate formulating policies and management strategies to mitigate HRQoL declines in $\mathrm{HBV}^{+}$patients.

\section{Abbreviations}

HBV: Hepatitis B virus; HRQoL: Health-related quality of life.

\section{Competing interests}

The authors declare that they have no competing interests.

\section{Authors' contributions}

SJK had the idea for the study, carried out the statistical analysis, interpreted the data and drafted the manuscript. ECP advised on the design of the study and the conception of the paper. KTH substantially contributed to the interpretation of the data and drafting the paper. SYL substantially contributed to drafting the paper. All authors read and approved the final manuscript.

\section{Acknowledgements}

None.

\section{Author details}

'Department of Public Health, Graduate School, Yonsei University, Seoul, Republic of Korea. ${ }^{2}$ Institute of Health Services Research, Yonsei University, Seoul, Republic of Korea. ${ }^{3}$ Department of Health Policy and Management, Graduate School of Public Health, Yonsei University, Seoul, Republic of Korea. ${ }^{4}$ Department of Preventive Medicine, Yonsei University College of Medicine, 50 Yonsei-ro, Seodaemun-gu, Seoul 120-752, Republic of Korea.

Received: 12 November 2014 Accepted: 29 April 2015

Published online: 12 May 2015

\section{References}

1. World Health Organization (WHO). Global Action Plan for the prevention and control of noncommunicable diseases 2013-2020. [http://www.who.int/ nmh/publications/ncd-action-plan/en/]

2. Lozano R, Naghavi M, Foreman K, Lim S, Shibuya K, Aboyans V, et al. Global and regional mortality from 235 causes of death for 20 age groups in 1990 and 2010: a systematic analysis for the Global Burden of Disease Study 2010. Lancet. 2012;380:2095-128.

3. Korea Center for Disease Control and Prevention: Korea Health Statics 2013: Cause of death. 2014. [http://www.index.go.kr/potal/main/ EachDtIPageDetail.do?idx_cd=2770]

4. Yeo Y, Gwack J, Kang S, Koo B, Jung SJ, Dhamala P, et al. Viral hepatitis and liver cancer in Korea: an epidemiological perspective. Asian Pacific journal of cancer prevention : APJCP. 2013;14:6227-31.

5. World Health Oranization (WHO). Global policy report on the prevention and control of viral hepatitis. 2013. [http://www.who.int/hiv/pub/hepatitis/ global_report/en/]

6. Kim WR. Epidemiology of hepatitis B in the United States. Hepatology. 2009:49:S28-34.

7. Philbin MM, Erby LA, Lee S, Juon HS. Hepatitis B and liver cancer among three Asian American sub-groups: a focus group inquiry. J Immigr Minor Health. 2012;14:858-68.

8. Elizabeth W, Hwang M, Ramsey Cheung MD. Global Epidemiology of Hepatitis B Virus (HBV) Infection. N Am J Med Sci. 2011;4:7-13. 
9. Kim H, Shin AR, Chung HH, Kim MK, Lee JS, Shim JJ, et al. Recent trends in hepatitis B virus infection in the general Korean population. Korean J Intern Med. 2013;28:413-9.

10. Kang J, Cho JH, Suh CW, Lee DH, Oh HB, Sohn YH, et al. High prevalence of hepatitis $B$ and hepatitis $C$ virus infections in Korean patients with hematopoietic malignancies. Ann Hematol. 2011;90:159-64.

11. Weinstein AA, Kallman Price J, Stepanova M, Poms LW, Fang Y, Moon J, et al. Depression in patients with nonalcoholic fatty liver disease and chronic viral hepatitis B and C. Psychosomatics. 2011;52:127-32.

12. Hsu PC, Federico CA, Krajden M, Yoshida EM, Bremner KE, Anderson FH, et al. Health utilities and psychometric quality of life in patients with early- and late-stage hepatitis C virus infection. J Gastroenterol Hepatol. 2012;27:149-57.

13. Strauss E, Dias Teixeira MC. Quality of life in hepatitis C. Liver Int. 2006:26:755-65.

14. Tillmann HL, Wiese M, Braun Y, Wiegand J, Tenckhoff S, Mossner J, et al. Quality of life in patients with various liver diseases: patients with HCV show greater mental impairment, while patients with PBC have greater physical impairment. J Viral Hepat. 2011;18:252-61.

15. Qureshi MO, Khokhar N, Shafqat F. Severity of depression in hepatitis B and hepatitis C patients. J Coll Phys Surg Pakistan. 2012;22:632-4.

16. Chang SC, Yang SS, Chang CC, Lin CC, Chung YC, Li TC. Assessment of health-related quality of life in antiviral-treated Taiwanese chronic hepatitis C patients using SF-36 and CLDQ. Health Qual Life Outcomes. 2014;12:97.

17. Zhuang G, Zhang M, Liu Y, Guo Y, Wu Q, Zhou K, et al. Significant impairment of health-related quality of life in mainland Chinese patients with chronic hepatitis B: a cross-sectional survey with pair-matched healthy controls. Health Qual Life Outcomes. 2014;12:101.

18. Levy AR, Kowdley KV, lloeje U, Tafesse E, Mukherjee J, Gish R, et al. The impact of chronic hepatitis B on quality of life: a multinational study of utilities from infected and uninfected persons. Value Health. 2008;11:527-38.

19. Bondini S, Kallman J, Dan A, Younoszai Z, Ramsey L, Nader F, et al. Health-related quality of life in patients with chronic hepatitis B. Liver Int. 2007;27:1119-25.

20. Haq N, Hassali MA, Shafie AA, Saleem F, Aljadhey H. A cross sectional assessment of health related quality of life among patients with Hepatitis-B in Pakistan. Health Qual Life Outcomes. 2012;10:91.

21. Lam ET, Lam CL, Lai CL, Yuen MF, Fong DY, So TM. Health-related quality of life of Southern Chinese with chronic hepatitis B infection. Health Qual Life Outcomes. 2009;7:52.

22. Kim HC, Kim BK, Min KB, Min JY, Hwang SH, Park SG. Association between job stress and insomnia in Korean workers. J Occup Health. 2011;53:164-74.

23. Park SG, Min KB, Chang SJ, Kim HC, Min JY. Job stress and depressive symptoms among Korean employees: the effects of culture on work. Int Arch Occup Environ Health. 2009;82:397-405.

24. Ong SC, Mak B, Aung MO, Li SC, Lim SG. Health-related quality of life in chronic hepatitis B patients. Hepatology. 2008;47:1108-17.

25. Woo G, Tomlinson G, Yim C, Lilly L, Therapondos G, Wong DK, et al. Health state utilities and quality of life in patients with hepatitis B. Can J Gastroenterol. 2012;26:445-51.

26. Chao J, Song $L$, Zhang $H$, Zhu L, Tian $L$, Jin $H$, et al. Effects of comprehensive intervention on health-related quality of life in patients with chronic hepatitis B in China. BMC Health Serv Res. 2013;13:386.

27. Quittner AL, Schechter MS, Rasouliyan L, Haselkorn T, Pasta DJ, Wagener JS Impact of socioeconomic status, race, and ethnicity on quality of life in patients with cystic fibrosis in the United States. Chest. 2010;137:642-50.

28. Wieclaw J, Agerbo E, Mortensen PB, Bonde JP. Occupational risk of affective and stress-related disorders in the Danish workforce. Scand J Work Environ Health. 2005;31:343-51

29. Park JH, Park JH, Kim SG, Lee KS, Hahm MI. Changes in employment status and experience of discrimination among cancer patients: findings from a nationwide survey in Korea. Psychooncology. 2010;19:1303-12.

30. Newgard CD, Hedges JR, Arthur M, Mullins RJ. Advanced statistics: the propensity score-a method for estimating treatment effect in observational research. Acad Emerg Med. 2004;11:953-61.

31. $H u K Q$, Pan $C Q$, Goodwin D. Barriers to screening for hepatitis $B$ virus infection in Asian Americans. Dig Dis Sci. 2011;56:3163-71.
32. Jung CW, Tan J, Tan N, Kuo MN, Ashok A, Eells SJ, et al. Evidence for the insufficient evaluation and undertreatment of chronic hepatitis B infection in a predominantly low-income and immigrant population. J Gastroenterol Hepatol. 2010;25:369-75.

33. Cohen C, Holmberg SD, McMahon BJ, Block JM, Brosgart CL, Gish RG, et al. Is chronic hepatitis B being undertreated in the United States? J Viral Hepat. 2011;18:377-83.

\section{Submit your next manuscript to BioMed Central and take full advantage of:}

- Convenient online submission

- Thorough peer review

- No space constraints or color figure charges

- Immediate publication on acceptance

- Inclusion in PubMed, CAS, Scopus and Google Scholar

- Research which is freely available for redistribution 